\title{
Enamel interproximal reduction during treatment with clear aligners: digital planning versus OrthoCAD analysis
}

\author{
Giuseppina Laganà, Arianna Malara*, Roberta Lione, Carlotta Danesi, Simonetta Meuli and Paola Cozza
}

\begin{abstract}
Background: The aim of the study was to compare the amount of interproximal enamel reduction (IPR) provided on ClinCheck software with the amount of IPR carried out by the orthodontist during treatment with clear aligners.

Methods: 30 subjects (14 males, 16 females; mean age of $24.53 \pm 13.41$ years) randomly recruited from the Invisalign account of the Department of Orthodontics at the University of Rome "Tor Vergata" from November 2018 to October 2019, were collected according to the following inclusion criteria: mild to moderate dento-alveolar discrepancy (1.5$6.5 \mathrm{~mm}$ ); Class I canine and molar relationship; full permanent dentition (excluding third molars); both arches treated only using Comprehensive Package by Invisalign system; treatment plan including IPR. Pre- (T0) and post-treatment (T1) digital models (.stl files), created from an iTero scan, were collected from all selected patients. The OrthoCAD digital software was used to measure tooth mesiodistal width in upper and lower arches before (TO) and at the end of treatment (T1) before any refinement. The widest mesio-distal diameter was measured for each tooth excluding molars by "Diagnostic" OrthoCAD tool. The total amount of IPR performed during treatment was obtained comparing the sum of mesio-distal widths of all measured teeth at T0 and T1. Significant T1-T0 differences were tested with dependent sample t-test $(P<0.05)$.
\end{abstract}

Results: In the upper arch, IPR was digitally planned on average for $0.62 \mathrm{~mm}$ while in the lower arch was on average for $1.92 \mathrm{~mm}$. As for the amount of enamel actually removed after IPR performing, it was on average $0.62 \mathrm{~mm}$ in the maxillary arch. In the mandibular arch, the mean of IPR carried out was $1.93 \mathrm{~mm}$. The difference between planned IPR and performed IPR is described: this difference was on average $0.00 \mathrm{~mm}$ in the upper arch and 0.01 in the lower arch.

Conclusions: The amount of enamel removed in vivo corresponded with the amount of IPR planned by the Orthodontist using ClinCheck software.

Keywords: Dental crowding, Interproximal enamel reduction, Invisalign, ClinCheck

\section{Background}

The Invisalign appliance was introduced for the first time to the public in the late 1990s by Align Technology as an innovative method to straightening teeth without braces [1]. This system uses impressions or intraoral scans

*Correspondence: drariannamalara@gmail.com

Department of Systems Medicine, University of Rome'Tor Vergata', Viale Oxford 81, 00133 Rome, Italy which are converted through stereolithographic technology (.stl) into virtual models and then launched with the ClinCheck software: a three-dimensional modeling program that allows a virtual simulation of teeth movements. A series of aligners is then produced in order to gain the needed corrections [2].

Most of patients treated with Invisalign present dental crowding that is a key reason for people seeking orthodontic treatment. Enamel interproximal reduction (IPR), 
extractions, increase in arch perimeter with distalization, buccal arch expansion, or incisor protrusion are important therapy strategies to solve dental crowding [3]. The main target of an orthodontic treatment is to provide the best balance among occlusal relationships, dental and facial esthetics and long-term treatment stability. Achieving these goals could be difficult in most patients because of the excess of tooth structures that often can interfere with the correct alignment of the teeth in the dental arch [4]. In cases of mild to moderate dental crowding, IPR represents a good therapy solution. It is a clinical procedure that allows to gain space in order to align teeth through the reduction, the anatomic recontouring and the protection of interproximal enamel surfaces of permanent teeth [5]. This technique may help achievement of treatment goals while preserving the integrity of the dental and periodontal tissues [6]. In overall terms, a major contribution of IPR is that the extent of expansion in the labial direction can be reduced, thus reducing the risk of bone dehiscence [7]. In additional, enlarged proximal contacts stabilize the treatment result [8]. IPR also provides to the elimination of "black triangles" allowing an improvement in the aesthetic appearance [7]. Creating the proper apposition areas for the gingiva also reduces or prevents retrusion of the interdental papillae [9]. In Invisalign treatment IPR is pre-planned during ClinCheck development: the clinician can decide the amount of IPR to be performed and the area and stage where it is needed [5]. To prevent residue excessive space, persisting misaligned teeth, or inter-arch discrepancies, it is important to quantify the amount of enamel that can be removed [10]. Moreover, the amount of IPR actually done has to be as the expected and programmed one in order to obtain the planned movements [11].

This high accuracy is essential to reach treatment objectives particularly during $3 \mathrm{D}$ digitally treatment plans [10].

Despite the available literature relating Invisalign technology, IPR reliability has been analyzed less deeply and the results still remains unclear. Although many studies have focused on the surface irregularities that could remain after grinding and polishing $[12,13]$, only a few papers presenting a quantitative evaluation of stripped enamel have been identified [7, 14]. Johner et al. aimed to investigate the predictability of the expected amount of IPR using three different methods on premolars. The study showed large variations in the amount of enamel removed, in fact, in most cases, actual stripping was on average less than the expected amount of enamel reduction and the different stripping technique was not significant [14]. Recently De Felice et al. investigated the fidelity of the IPR performed by manually system, during clear aligners therapy, and showed that the amount of enamel removed in vivo did not correspond to the expected amount of IPR [11].

In this regard, the aim of the present study was to evaluate, in terms of enamel reduction, the accuracy and the correspondence of the IPR planned by ClinCheck software and the IPR carried out by the orthodontist by mechanical oscillating systems. The null hypothesis was that there is not a difference between the expected and the actual amounts of enamel reduction.

\section{Methods}

This study followed the principles laid down by the World Medical Assembly in the Declaration of Helsinki 2008 on medical protocols and ethics and received positive response by the Ethic Committee at the University of Rome Tor Vergata (protocol number: 141/19). For the present retrospective study 30 subjects (14 males, 16 females; mean age of $24.53 \pm 13.41$ years) randomly recruited from the Invisalign account of the Department of Orthodontics at the University of Rome "Tor Vergata" from November 2018 to October 2019, were collected according to the following inclusion criteria:

- mild to moderate dento-alveolar discrepancy (1.5$6.5 \mathrm{~mm}$ );

- Class I canine and molar relationship;

- full permanent dentition (excluding third molars);

- both arches treated only using Comprehensive Package by Invisalign system;

- treatment plan including IPR.

Patients with dentofacial deformity or medical problems, poor compliance with aligners, extractions therapy, auxiliaries other than Invisalign attachments, Invisalign no Comprehensive Package therapy, multiple and/or advanced caries, impacted, missing, or supernumerary teeth, prosthetic restorations, were excluded. Written consent was obtained from all participating subjects.

For the treatment protocol, each subject was instructed to wear aligners for $22 \mathrm{~h}$ per day, except during meals and oral hygiene procedures and to replace aligners on average every 15 days. Every 6 stages the clinician checked the good aligner fitting and the position of the attachments. The mean number of aligners was 25 for the maxillary arch and 24 for the mandibular arch. Both arches averaged 8 attachments and less than $2.5 \mathrm{~mm}$ of IPR in the upper arch and less than $4.5 \mathrm{~mm}$ of IPR in the lower arch.

IPR was carried out by the same experienced operator (G.L.) at the programmed phase according to the virtual treatment staging under water-cooling. It was planned by ClinCheck software, from the mesial surface of the right second premolar to the mesial surface of the left second 
premolar in both arches, according to treatment needs of the single clinical case and then recorded in an Excel file. IPR was achieved using oscillating abrasive strip mounted in a contra-angle handpiece with rotation speed of 20,000 rpm as suggested by the manufacturer and the amount of space obtained was checked with metal gages. To amplify the remineralization capacity of the abraded proximal surfaces, a topical fluoride gel was applied on the reduced teeth for $5 \mathrm{~min}$.

Pre- (T0) and post-treatment (T1) digital models (.stl files), created from an iTero scan, were collected from all selected patients.

\section{Data measurement}

The pre-treatment and post-treatment .stl files were uploaded into OrthoCAD software (3Shape, Copenhagen, Denmark) version 5.9.0.36. The OrthoCAD digital software was used to measure tooth mesiodistal width in upper and lower arches before (T0) and at the end of treatment (T1) before any refinement (Fig. 1). The widest mesio-distal diameter was measured for each tooth excluding molars by "Diagnostic" OrthoCAD tool. The total amount of IPR performed during treatment was obtained comparing the sum of mesio-distal widths of all measured teeth at $\mathrm{T} 0$ and $\mathrm{T} 1$.

To determine the reliability of the method, all measurements were made by a single operator (A.M.) who was blinded to the entity of IPR planned and performed, and were checked by a second operator (S.M.). They were repeated 2 weeks after the first assessment. A paired $t$-test was used to compare the two measurements (systematic error). The magnitude of the random error was calculated by using the method of moment's estimator [15].

\section{Statistical analysis}

The power of the study for the independent sample $t$-test was calculated on the basis of the sample size of two groups and an effect size equal to 0.9 [16]. The power was 0.80 at an alpha level of 0.05 (SigmaStat 3.5, Systat Software, Point Richmond, California, USA).

In the presence of normally distributed data, a paired $t$-test was selected to compare the T1-T0 changes. The level of significance was set at $5 \%$.

SPSS (Statistical Package for the Social Sciences), version 18.0 (IBM Corp, Chicago III) was the chosen software to analyze data.

\section{Results}

No systematic error was found among the repeated digital measurements. The systematic error was reduced by precise definitions of points in the presence of a previously trained experienced examiner. The mean random error was $0.42 \mathrm{~mm}$ and within acceptable limits because the software allowed a more accurate view of the anatomic details. The mean time between the initial and the final scans was 11.87 months.

47 arches were collected for this investigation: in 17 patients interproximal enamel reduction was digitally planned and performed in both arches, in 2
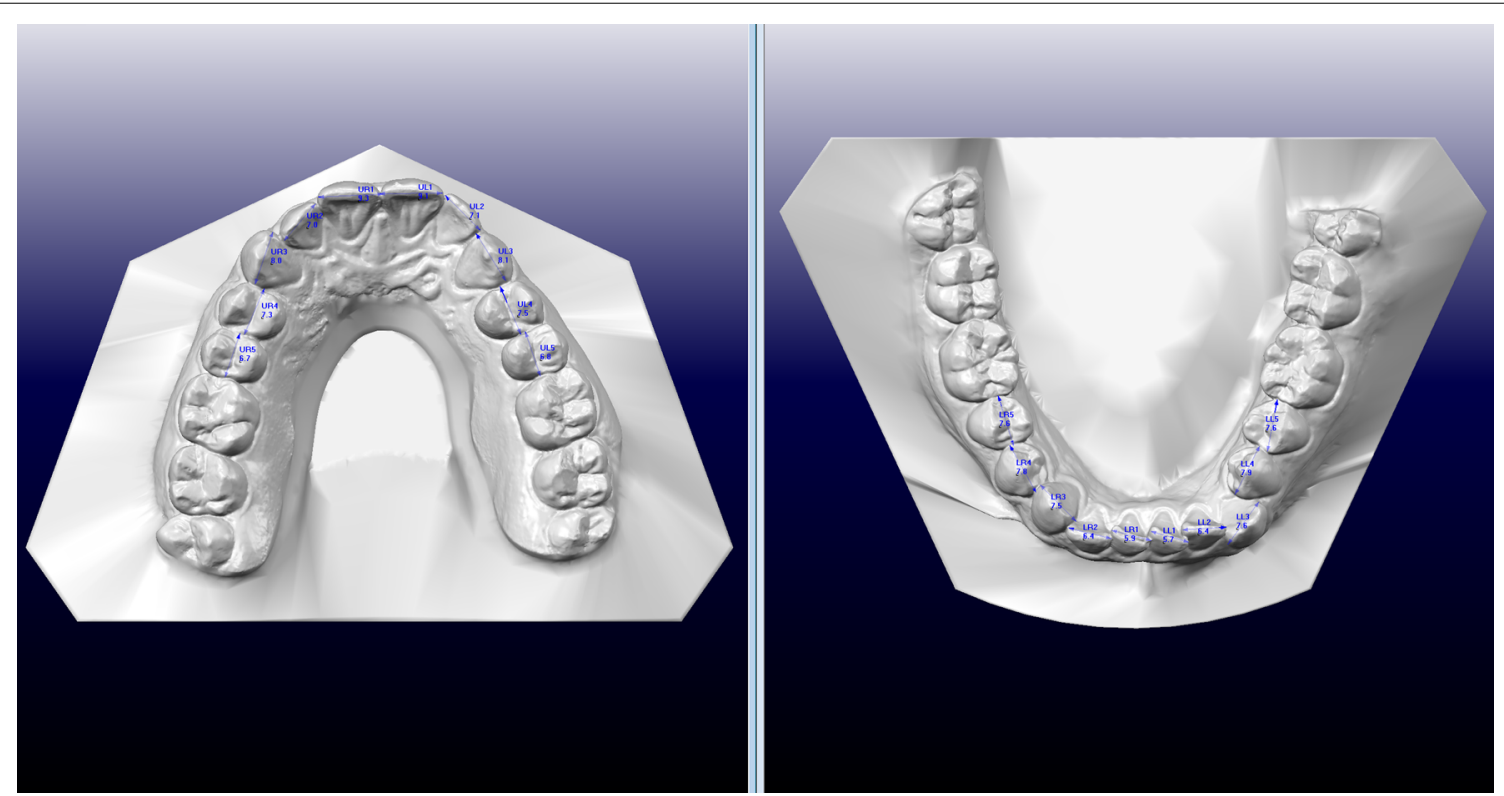

Fig. 1 Mesio-distal diameters analysis by OrthoCAD software before the treatment (T0) 
subjects only in the maxillary arch while in 11 participants only in the mandibular arch. The mean of IPR digitally planned in the upper arch was $0.62 \mathrm{~mm}$ with values ranging from 0.20 to $2.20 \mathrm{~mm}$, in the lower arch it was $1.92 \mathrm{~mm}$ with values ranging from 0.20 to $4.40 \mathrm{~mm}$ (Table 1). As for the amount of enamel actually removed after IPR performing, it was on average $0.62 \mathrm{~mm}$ in the maxillary arch. In the mandibular arch, the mean of IPR carried out was $1.93 \mathrm{~mm}$ (Table 1).

In the Table 2 the difference between planned IPR and performed IPR is described: this difference was on average $0.00 \mathrm{~mm}$ in the upper arch and 0.01 in the lower arch.

Table 1 Descriptive analysis of the study group

\begin{tabular}{|c|c|}
\hline Study gr & \\
\hline Age (year & \\
\hline Mean & 24.53 \\
\hline Min & 11 \\
\hline Max & 56 \\
\hline Gender & \\
\hline M & 14 \\
\hline $\mathrm{F}$ & 16 \\
\hline Upper ar & \\
\hline IPR planr & \\
\hline Mean & 0.62 \\
\hline Min & 0.20 \\
\hline Max & 2.20 \\
\hline IPR perfo & \\
\hline Mean & 0.62 \\
\hline Min & 0.20 \\
\hline Max & 2.30 \\
\hline Lower ar & \\
\hline IPR planr & \\
\hline Mean & 1.92 \\
\hline Min & 0.20 \\
\hline Max & 4.40 \\
\hline IPR perfo & \\
\hline Mean & 1.93 \\
\hline Min & 0.20 \\
\hline Max & 4.50 \\
\hline
\end{tabular}

No statistically significant differences were found in both upper and in lower arch.

\section{Discussion}

The purpose of the present study was to verify, through a three-dimensional data set, the accuracy and the correspondence between the IPR provided by ClinCheck software and the IPR carried out by the orthodontist by mechanical oscillating systems. Therefore, careful preoperative planning by using ClinCheck software is essential to determine where and when to perform IPR and its amount in order to achieve the expected results. IPR and staging are important items to manage on each and every patient. Proper IPR technique and staging of events such as new attachment placement can help ensure treatment proceeds smoothly and appropriately [17]. This study focused on the use of the oscillating abrasive strip mounted in a contra-angle handpiece to perform IPR. Oscillating strips are easy to use and provide precise enamel reduction, better visualization and access. They are available in several widths and are color-coded, offering control over specific point-of-contact reduction. IPR should be performed incrementally, proceeding through increasing widths to the desired amount rather than selecting the thickest width first. This modality is particularly useful in the anterior segments because of the ease of access. It is important not to use excessive pressure to avoid the risk of strip fracture and enamel surface protrusions [18].

IPR plays an important role in non-extraction treatment to obtain space to align teeth and/ or to achieve more long-term alignment stability [6]. So, knowledge of the predictability of this procedure is important to improve treatment outcomes for this technique. Nevertheless, there are very few studies in literature dealing with this purpose $[7,11,14]$. Surely the most important one, as well as recent and similar to ours is by De Felice et al. [11] whose main target was to compare the accuracy of the actual space obtained through interproximal enamel reduction to the amount of IPR planned through the digital setup during clear aligner treatment. Although the present study showed a concordance between planned and actually performed IPR, De Felice et al. demonstrated that the amount of enamel removed in vivo did not correspond with the amount of IPR

Table 2 Descriptive statistics and statistical comparison between T0 and T1 differences by means of Student $t$-test for paired groups

\begin{tabular}{|c|c|c|c|c|c|}
\hline & $\begin{array}{l}\text { Mean of IPR digitally planned } \\
\text { (T0) (mm) }\end{array}$ & $\begin{array}{l}\text { Mean of IPR performed (T1) } \\
(\mathrm{mm})\end{array}$ & T1-T0 & $95 \% \mathrm{Cl}$ & $p$ values \\
\hline Upper arch & $0.62 \pm 0.63$ & $0.62 \pm 0.63$ & 0.00 & -0.16 to 0.16 & NS \\
\hline Lower arch & $1.92 \pm 1.93$ & $1.93 \pm 1.97$ & 0.01 & -0.18 to 0.12 & NS \\
\hline
\end{tabular}

NS not significant, $\mathrm{Cl}$ confidence interval 
planned and, indeed, in most cases the amount of IPR performed was lower. This difference can be attributed to two key factors. First, the IPR technique. De Felice et al. used the manual method with single-sided diamondcoated strips; in our study, instead, the stripping method consisted of a mechanical oscillating system. This might suggest a greater predictability of the amount of IPR and of the mechanical method that, anyway, was not the aim of the present study.

However, the greater efficacy and precision of IPR the mechanical method compared to the manual one is reported in literature by different papers $[10,13,19]$. Mechanical strips have been shown to be more efficient in reducing enamel than the manual system; in particular, the mechanical system reduced the inaccuracy of the manual one satisfying precision potentially down to $0.1 \mathrm{~mm}$ required by $3 \mathrm{D}$ treatment planes such as clear aligners [10]. The decrease in abrasive properties is significantly less considerable for mechanical IPR system and, as a matter of fact, according to the recent survey by Kaaouara et al. [20], the mechanical oscillating diamond strips produced a more regular surface, with light parallel lines and smaller grooves than manual abrasive strips, although a constant decrease has been observed for both systems [21].

Finally, another substantial difference can be found in the recruitment of the subjects in the study group. In our investigation, the patients were selected from a single Invisalign provider, while in the study by De Felice et al. the subjects were selected from ten different orthodontists. As it is recognized in literature, the amount of enamel removed by stripping is influenced by operator or technique factors including the pressure applied, the hardness, the size of the abrasive, the duration of IPR, and tooth-related aspects such as enamel hardness [22]. In order to guarantee standardization of the experimental IPR technique, in our study it was performed by a single clinician within a pre-established period, while closely following the manufacturers' instructions [23].

\section{Conclusions}

The null hypothesis of the study is confirmed: the amount of enamel removed in vivo corresponds with the amount of IPR planned by the Orthodontist using ClinCheck software. This result could be due to the IPR method and to the high experience of the Orthodontist who performed it, as a matter of fact IPR is influenced by operator or technique factors [22].

\section{Acknowledgements}

Not applicable.

\section{Authors' contributions}

AM and CD performed the experimental analysis, RL analyzed the data. GL performed IPR and contributed to write the manuscript, SM performed and tested OrthoCAD measuring method. PC supervised the project and contributed in writing the manuscript. All authors have read and approved the manuscript.

\section{Funding}

No funding was provided.

\section{Availability of data and materials}

The datasets used and/or analysed during the current study are available from the corresponding author on reasonable request.

\section{Ethics approval and consent to participate}

This study followed the principles laid down by the World Medical Assembly in the Declaration of Helsinki 2008 Helsinki Declarations on medical protocols and ethics and received positive response by the Ethic Committee at the University of Rome Tor Vergata (Protocol No. 141/19). Written informed consent was obtained from all the participants for the study.

\section{Consent for publication}

Not applicable.

\section{Competing interests}

The authors declare that they have no competing interests.

Received: 15 September 2020 Accepted: 25 February 2021

Published online: 19 April 2021

\section{References}

1. Charalampakis $\mathrm{O}$, Iliadi A, Ueno H, Oliver DR, Kim KB. Accuracy of clear aligners: a retrospective study of patients who needed refinement. Am J Orthod Dentofac Orthop. 2018;154(1):47-54.

2. Lanteri V, Farronato G, Lanteri C, Caravita R, Cossellu G. The efficacy of orthodontic treatments for anterior crowding with Invisalign compared with fixed appliances using the Peer Assessment Rating Index. Quintessence Int. 2018;49(7):581-7.

3. Duncan LO, Piedade L, Lekic M, Cunha RS, Wiltshire WA. Changes in mandibular incisor position and arch form resulting from Invisalign correction of the crowded dentition treated nonextraction. Angle Orthod. 2016;86(4):577-83.

4. Lapenaite $E$, Lopatiene K. Interproximal enamel reduction as a part of orthodontic treatment. Stomatologija. 2014;16(1):19-24.

5. Kravitz ND, Kusnoto B, BeGole E, Obrez A, Agran B. How well does Invisalign work? A prospective clinical study evaluating the efficacy of tooth movement with Invisalign. Am J Orthod Dentofac Orthop. 2009;135(1):27-35.

6. Livas $C$, Jongsma AC, Ren Y. Enamel reduction techniques in orthodontics: a literature review. Open Dent J. 2013;7:146-51.

7. Hellak A, Schmidt N, Schauseil M, Stein S, Drechsler T, Korbmacher-Steiner $\mathrm{HM}$. Influence on interradicular bone volume of Invisalign treatment for adult crowding with interproximal enamel reduction: a retrospective three-dimensional cone-beam computed tomography study. BMC Oral Health. 2018;18(1):103.

8. Allais D, Melsen B. Does labial movement of lower incisors influence the level of the gingival margin? A case-control study of adult orthodontic patients. Eur J Orthod. 2003;25(4):343-52.

9. Melsen B, Allais D. Factors of importance for the development of dehiscences during labial movement of mandibular incisors: a retrospective study of adult orthodontic patients. Am J Orthod Dentofac Orthop. 2005;127(5):552-61.

10. Gazzani F, Lione R, Pavoni C, Mampieri G, Cozza P. Comparison of the abrasive properties of two different systems for interproximal enamel reduction: oscillating versus manual strips. BMC Oral Health. 2019;19(1):247.

11. De Felice ME, Nucci L, Fiori A, Flores-Mir C, Perillo L, Grassia V. Accuracy of interproximal enamel reduction during clear aligner treatment. Prog Orthod. 2020;21(1):28. 
12. Zhong M, Jost-Brinkmann PG, Zellmann M, Zellmann S, Radlanski RJ. Clinical evaluation of a new technique for interdental enamel reduction. J Orofac Orthop. 2000;61:432-9.

13. Danesh G, Hellak A, Lippold C, Ziebura T, Schafer E. Enamel surfaces following interproximal reduction with different methods. Angle Orthod. 2007;77(6):1004-10

14. Johner AM, Pandis N, Dudic A, Kiliaridis S. Quantitative comparison of 3 enamel-stripping devices in vitro: how precisely can we strip teeth? Am J Orthod Dentofac Orthop. 2013;143(4 Suppl):S168-72.

15. Springate SD. The effect of sample size and bias on the reliability of estimates of error: a comparative study of Dahlberg's formula. Euro J Orthod. 2012;34:158-63.

16. Cohen J. A power primer. Psychol Bull. 1992;112:155-9.

17. Glazer B. The Insider's Guide to Invisalign Treatment: A Step-By-step Guide to Assist You with Your ClinCheck Treatment Plans. 3L Publishing, 2017.

18. Pindoria J, Fleming PS, Sharma PK. Inter-proximal enamel reduction in contemporary orthodontics. Br Dent J. 2016;221(12):757-63.

19. Piacentini C, Sfondrini G. A scanning electron microscopy comparison of enamel polishing methods after air-rotor stripping. AJODO. 1996;109:57-63.
20. Kaaouara Y, Mohind HB, Azaroual MF, Zaoui F, Bahije L, Benyahia H. In vivo enamel stripping: a macroscopic and microscopic analytical study. Int Orthod. 2019;17(2):235-42.

21. Lione R, Gazzani F, Pavoni C, Guarino S, Tagliaferri V, Cozza P. In vitro and in vivo evaluation of diamond-coated strips. Angle Orthod. 2017:87(3):455-9.

22. Hein $C$, Jost-Brinkmann PG, Schillai G. The enamel surface quality after interproximal stripping - a scanning electron microscopic assessment of different polishing procedures. Fortschr Kieferorthop. 1990;51:327-35.

23. Livas C, Baumann T, Flury S, Pandis N. Quantitative evaluation of the progressive wear of powered interproximal reduction systems after repeated use: an in vitro study. J Orofac Orthop. 2020;81(1):22-9.

\section{Publisher's Note}

Springer Nature remains neutral with regard to jurisdictional claims in published maps and institutional affiliations.
Ready to submit your research? Choose BMC and benefit from:

- fast, convenient online submission

- thorough peer review by experienced researchers in your field

- rapid publication on acceptance

- support for research data, including large and complex data types

- gold Open Access which fosters wider collaboration and increased citations

- maximum visibility for your research: over $100 \mathrm{M}$ website views per year

At BMC, research is always in progress.

Learn more biomedcentral.com/submissions 